\title{
Beam dynamics in Compton ring gamma sources
}

\author{
Eugene Bulyak, ${ }^{*}$ Peter Gladkikh, and Vladislav Skomorokhov \\ NSC KIPT, Kharkov, Ukraine \\ Tsunehiko Omori and Junji Urakawa \\ KEK, Ibaraki, Japan \\ Klaus Moenig \\ DESY, Zeuthen, Germany, and LAL, Orsay, France
}

Frank Zimmermann

CERN, Geneva, Switzerland

(Received 17 August 2006; published 18 September 2006)

\begin{abstract}
Electron storage rings of $\mathrm{GeV}$ energy with laser pulse stacking cavities are promising intense sources of polarized hard photons which, via pair production, can be used to generate polarized positron beams. In this paper, the dynamics of electron bunches circulating in a storage ring and interacting with high-power laser pulses is studied both analytically and by simulation. Both the common features and the differences in the behavior of bunches interacting with an extremely high power laser pulse and with a moderate pulse are discussed. Also considerations on particular lattice designs for Compton gamma rings are presented.
\end{abstract}

DOI: 10.1103/PhysRevSTAB.9.094001

PACS numbers: 29.27.Bd, 29.20.-c, 41.75.Ht

\section{INTRODUCTION: WHAT ARE COMPTON GAMMA SOURCES?}

We refer to the notion "Compton gamma source" as an electron ring equipped with a powerful laser system able to generate polarized photons at an energy of a few tens of $\mathrm{MeV}$ by scattering polarized laser photons off ultrarelativistic electrons; see [1,2]. The idea of storing electrons in a ring and laser pulses in an optical resonator suggested in [3] could overcome limitations imposed by a small electron-photon scattering cross section and enable the effective production of intense beams of high-energy photons from moderate-energy electrons.

It is planned to employ such sources for the generation of intense bunches of highly polarized positrons at the future linear-collider projects ILC [4] and CLIC [5].

The electron beam dynamics in Compton gamma sources is similar to that in Compton $\mathrm{x}$-ray sources [3,68]. Therefore, in this paper, we will first recall some basic facts about the beam dynamics in x-ray sources. Then, we will emphasize the distinctions which arise from the main difference - a much larger energy of the circulating electrons in a gamma ring (gamma-ray energies may be several tens of $\mathrm{MeV}$, while $\mathrm{x}$-ray energies range from $100 \mathrm{eV}$ to $100 \mathrm{keV}$ ). Beam-dynamics simulation results for a gamma source are presented next. The report closes with a summary and outlook.

\footnotetext{
*Electronic address: bulyak@kipt.kharkov.ua
}

\section{BEAM DYNAMICS IN COMPTON X-RAY SOURCES}

In both analytical descriptions and simulations, the laser pulse is represented by a bunch of photon (macro)particles. The beam-dynamics study is based on the assumed equality of the recoil momentum received by an electron and the momentum of the scattered laser photon [9]. The small initial momentum of the laser photon is neglected. This corresponds to the Thomson limit of the Compton scattering cross section, namely, to the zeroth-order term in a power series expansion of the recoil (and spectrum) with respect to $\gamma E_{\text {las }} /\left(m_{e} c^{2}\right)$, which is of order $\approx 5 \times 10^{-3}$ for the YAG rings considered in this paper; above $E_{\text {las }}$ denotes the laser-photon energy, $\gamma$ the Lorentz factor of the electron beam, and $m_{e}$ the electron rest mass. Schematically the process of scattering between a laser photon and a circulating electron is depicted in Fig. 1.

The maximal energy in the spectrum of photons scattered off an ultrarelativistic electron is

$$
E_{x}^{\max }=2 \gamma^{2}(1+\cos \varphi) E_{\mathrm{las}}
$$

where $\varphi$ denotes the collision angle ( $\varphi=0$ corresponds to a head-on collision). Only two-particle collisions are taken into consideration; $\mathrm{cf}$. [1].

The recoil momentum vector has the random length $P_{\text {recoil }}$ and random angles $\phi$ and $\psi$ with respect to the initial electron momentum. The recoil of the electron scales with $\gamma^{2}$, namely, 


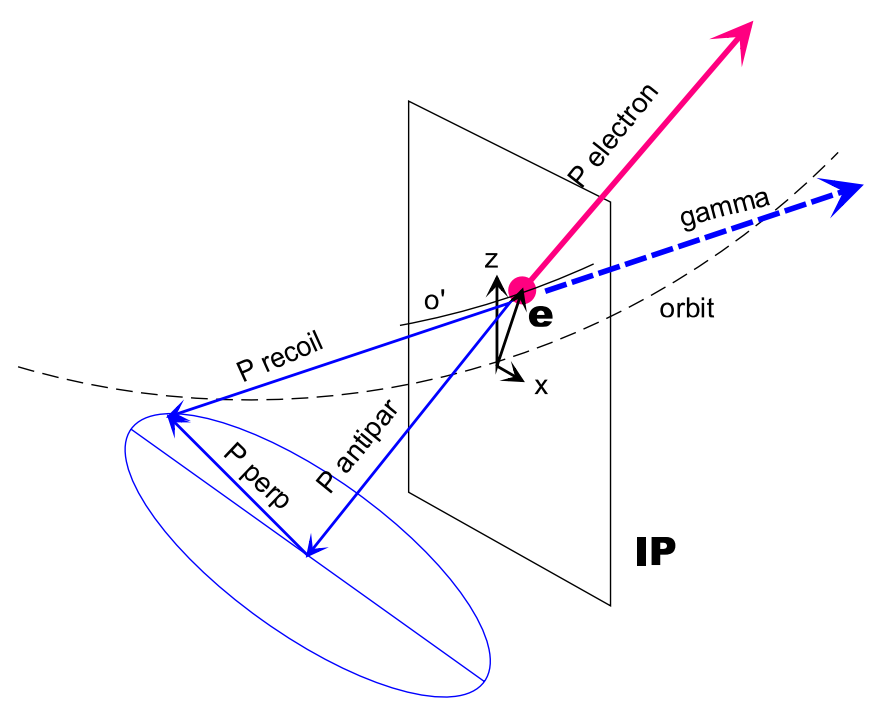

FIG. 1. (Color) Schematic of electron recoil in laser-photon Compton scattering.

$$
P_{\text {recoil }}=\frac{E_{x}^{\max } \zeta \gamma^{2}}{\gamma_{s}^{2} c}
$$

where $\gamma_{s}$ signifies the Lorentz factor of the synchronous particle, $\zeta$ the reduced energy of the gamma (the ratio of the energy of the scattered gamma to the maximal energy of gammas scattered off a synchronous electron with nominal energy $\zeta=E_{x} / E_{x}^{\max }$ ).

The spectral density of the reduced energy of gammas scattered off monoenergetic electrons is given by (see [10])

$$
F(\zeta)=\frac{3}{2}[1-2 \zeta(1-\zeta)] ; \quad 0 \leq \zeta \leq 1 .
$$

For ultrarelativistic electrons, the longitudinal kick is approximately equal to the momentum carried away by the photon.

The angle $\psi$ between the direction tangential to the electron trajectory and that of the recoil momentum depends on the absolute value of the recoil, i.e., the lateral kick $P_{\text {perp }}$ is a function of the recoil,

$$
P_{\text {perp }}=\frac{E_{x}^{\max }}{\gamma c} \sqrt{\zeta(1-\zeta)}
$$

The difference between the total kick (2) and the absolute value of its transverse component (4) should be emphasized: the total kick scales with the electron energy as $\gamma^{2}$, the transverse one as $\gamma$.

\section{A. Steady energy spread and transverse emittances}

$a$. The squared partial energy spread $s_{E}^{2}$ in Compton x-ray sources, i.e., the spread when the only Compton interactions take place, was analytically derived under the following approximations (see [9]): (i) linear momentum compaction factor; (ii) rare interaction rate (only a few electron-photon interactions per synchrotron-oscillation period; compare [11]); (iii) ring energy acceptance (separatrix height) sufficiently higher than the rms energy spread (5).

This spread reads

$$
s_{E}^{2} \equiv \frac{\sigma_{\gamma}^{2}}{\gamma_{s}^{2}}=\frac{7}{20} \frac{\gamma_{s}(1+\cos \varphi) E_{\mathrm{las}}}{m_{e} c^{2}},
$$

where $\sigma_{\gamma}$ is rms energy spread of the circulating electrons.

$b$. The transverse emittances are treated in a similar way. Assuming (i) uncoupled betatron motion without resonances; (ii) an interaction point (ip) located in a dispersion-free region; and (iii) minimum of beta functions at the ip; the partial emittance becomes (see [9])

$$
\epsilon_{c}=\frac{3 \beta_{\mathrm{ip}}}{10} \frac{E_{\mathrm{las}}}{\gamma_{s} m_{e} c^{2}},
$$

with $\beta_{\text {ip }}$ the beta function at the interaction point (ip). (The transverse emittance is defined as the rms trace-space emittance, $\boldsymbol{\epsilon}_{x}=\sqrt{\left\langle x^{2}\right\rangle\left\langle x^{\prime 2}\right\rangle-\left\langle x x^{\prime}\right\rangle^{2}}$ with $\quad x^{\prime}=P_{x} / P_{y}$ $[12,13]$.

If the electron bunch undergoes synchrotron excitation and damping, the resulting squared energy spread equals the weighted sum of the partial synchrotron and Compton energy spreads

$$
s^{2}=\frac{s_{\mathrm{sr}}^{2}(\Delta E)_{\mathrm{sr}}+s_{c}^{2}(\Delta E)_{c}}{(\Delta E)_{\mathrm{sr}}+(\Delta E)_{c}},
$$

where $(\Delta E)_{\mathrm{sr}, c}$ designates the partial energy losses per turn due to synchrotron radiation and Compton scattering, respectively.

The transverse emittances $\epsilon_{x, z}$ behave similarly to (7) with the substitution $s^{2} \rightarrow \epsilon_{x, z}$.

Usually, the energy spread due to Compton scattering exceeds the synchrotron-radiation one, while the transverse emittances may be larger or smaller than the natural (synchrotron) emittances depending on the beta-function value at the ip. It is also worth emphasizing that the squared energy spread increases with the energy of the electrons, whereas the transverse emittances decrease.

\section{B. Transition time}

The transition of the system from an arbitrary initial situation to the steady state can be derived under the assumption of rare interactions. In this case, the kinetic equation describing the evolution of the bunch can be formulated in terms of integrals of motion (BelyaevBudker equation [11]) and, thereby, reduced to the Ornstein-Uhlenbeck process. The transit times obtained obey the classical Kolomensky-Lebedev-Robinson theorem: (i) The longitudinal transition time (in unit of turns) is

$$
\tau_{c, \|}=\frac{E_{b}}{(\Delta E)_{c}}
$$

with $(\Delta E)_{\mathrm{c}}$ denoting the energy loss per turn due to 
Compton scattering, $E_{\mathrm{b}}$ the energy of the electrons. (ii) The transverse degrees of freedom change 2 times more slowly, i.e.,

$$
\tau_{c, \perp}=2 \tau_{c, \|}
$$

\section{BUNCH DYNAMICS IN COMPTON GAMMA RINGS}

The main difference between gamma-ray and x-ray rings arises from the following: (i) In gamma rings, the energy of the emitted photons of some tens of $\mathrm{MeV}$ is comparable to the available rf voltage; and (ii) the average number of interactions experienced by each electron per turn (per ip passage) needs to be high, varying from 0.03 for the CLIC positron source [2] to about unity for the ILC source [1], in order to provide the required total yield of polarized positrons.

Substituting the estimated parameters of a Compton gamma source of Table I (e.g., $E_{b}=1.3 \mathrm{GeV}$ for a ring with YAG lasers) into (5) and (6) reveals a large steadystate energy spread (about 6.4\% for YAG ring). At the same time the transverse emittances may easily reach the required values by using moderately low beta functions at the ip. Therefore, the longitudinal motion is the "bottleneck" in gamma rings.

Because of the large flux of gammas, the mirrors of the laser pulse stacking cavities must be placed outside of the gamma beam, and head-on electron-photon collisions should be avoided. For non-head-on interactions, the gamma yield crucially depends on the bunch length, so that the main problem of the ring design consists in keeping the bunch length as short as possible while interacting

TABLE I. YAG-laser Compton-source parameters for CLIC and ILC $[1,2]$.

\begin{tabular}{lcc}
\hline \hline \multicolumn{1}{c}{ Parameter } & CLIC & ILC \\
\hline Compton-ring energy & $1.3 \mathrm{GeV}$ & $1.3 \mathrm{GeV}$ \\
C-ring circumference & $42 \mathrm{~m}$ & $277 \mathrm{~m}$ \\
rf frequency & $1.875 \mathrm{GHz}$ & $650 \mathrm{MHz}$ \\
Bunch spacing & $0.16 \mathrm{~m}$ & $0.923 \mathrm{~m}$ \\
Number of bunches & 220 & 280 \\
Bunch population & $6.2 \times 10^{10}$ & $6.2 \times 10^{10}$ \\
Number of optical cavities & 1 & 30 \\
Total $\gamma^{\prime}$ s/bunch/turn & $2.8 \times 10^{9}$ & $5.8 \times 10^{10}$ \\
Selected $\gamma^{\prime}$ s/bunch/turn & $6.9 \times 10^{8}$ & $1.36 \times 10^{10}$ \\
Polarized $e^{+} /$bunch/turn & $9.8 \times 10^{6}$ & $1.9 \times 10^{8}$ \\
Injections/bunch & 300 & 100 \\
Total number injections & $6.6 \times 10^{4}$ & $2.8 \times 10^{5}$ \\
Number $e^{+} /$pulse & $6.5 \times 10^{11}$ & $5.3 \times 10^{13}$ \\
Number $e^{+} /$second & $9.7 \times 10^{13}$ & $2.7 \times 10^{14}$ \\
Number Compton-ring turns & 300 & 100 \\
Compton-pulse duration & $42 \mu \mathrm{s}$ & $90 \mu \mathrm{s}$ \\
Pause between cycles & $6.1 \mathrm{~ms}$ & $9.9 \mathrm{~ms}$ \\
\hline \hline
\end{tabular}

with the laser pulse. In the following, we consider a realistic crossing angle of 10 degrees.

\section{A. Pulsed mode of operation}

There exists another factor affecting the longitudinal dynamics: a large synchronous phase shift caused by the interaction with the laser pulses stored in the optical cavities. The synchronous phase angle $\phi_{s}$ is determined by the ratio of the average energy loss per turn $\Delta E_{b}$ to the peak energy gain from the rf system $e U_{\text {rf }}$ via

$$
\phi_{s}=\arcsin \left(\frac{\Delta E_{b}}{e U_{\mathrm{rf}}}\right)
$$

The interaction with the laser photons would shift the synchronous phase angle of the bunch center outside of the laser pulse, i.e.,

$$
\Delta l_{\text {bunch }}=\frac{\Delta \phi_{s} \lambda_{\mathrm{rf}}}{2 \pi} \approx \frac{c}{2 \pi f_{\mathrm{rf}}} \frac{\left(\Delta E_{C}-\Delta E_{\mathrm{s}}\right)}{U_{\mathrm{rf}}} \geq s_{\text {las }} .
$$

In addition, the positrons produced with the polarized gammas must be cooled in a damping ring as the linear colliders require tiny bunches at input. For economic reasons, the operation of the Compton gamma source should then also be pulsed.

All these considerations give rise to the idea of pulsed mode of operation of the gamma ring itself. In the pulsed mode, the laser system is switched on during a relatively short period of the operating cycle. The remaining time of the cycle is used for cooling down the Compton-ring electron bunches via synchrotron-radiation emission in the Compton-ring arcs and complementary wigglers.

The duration of gamma generation in the working cycle should be shorter than the time of the bunch degradation (unacceptable bunch-length increase or synchronous phase shift).

\section{B. Phase manipulation scheme}

The gamma yield in the pulsed mode of operation can be significantly enhanced if the rf-phase manipulation (RFPM) scheme is employed. The idea of the phase manipulation consists in providing interactions with the laser pulse at a shifted rf phase, different from the synchronous one - and exciting a coherent synchrotron oscillation before the gamma generation. In this mode, the phase in rf cavity is manipulated as follows (see Fig. 2): (1) fast ("shock") switch in rf phase; (2) "plateau" for quasisteady interaction with the laser; (3) slow adiabatic return into the initial position.

A simulated trajectory of the bunch centroid in the longitudinal phase space for this mode of operation is presented in Fig. 3 with the laser switched off. In this figure and in the following, the relative momentum deviation $p \equiv$ $\left(\gamma-\gamma_{s}\right) / \gamma_{s}$ is introduced as the momentum canonically conjugated with the rf phase of the particle $\phi$. 


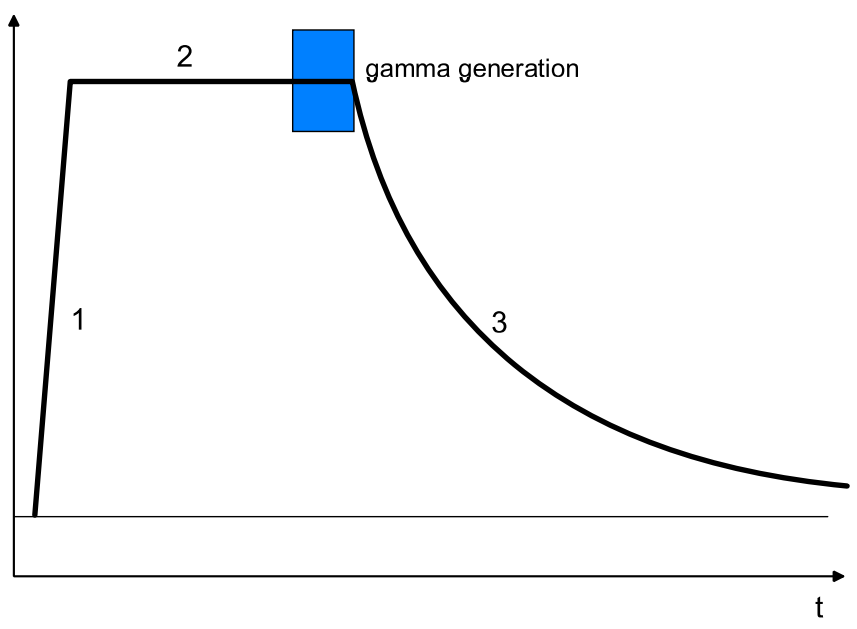

FIG. 2. (Color) The rf phase vs time.

\section{Nonlinear lattice}

The lattice of the gamma ring must provide a large energy acceptance for the circulating electron bunches. Since the acceptance ( $\mathrm{rf}$ separatrix height) is inversely proportional to the linear momentum compaction factor (more exactly, to the phase slipping factor [14]), this factor should be made as small as possible. The small momentum compaction corresponds to a small frequency of synchrotron oscillations: the particle abruptly loses energy over many successive turns while its longitudinal position hardly changes.

In the case of a small linear momentum compaction factor, higher orders in the compaction (phase slipping) come into play. As has been established (see, e.g., $[15,16]$ ), the second order factor leads to bunch deterioration. Thus, it should be eliminated. By contrast, the third-order momentum compaction factor plays a positive role: it can increase the gamma yield. It is especially important for

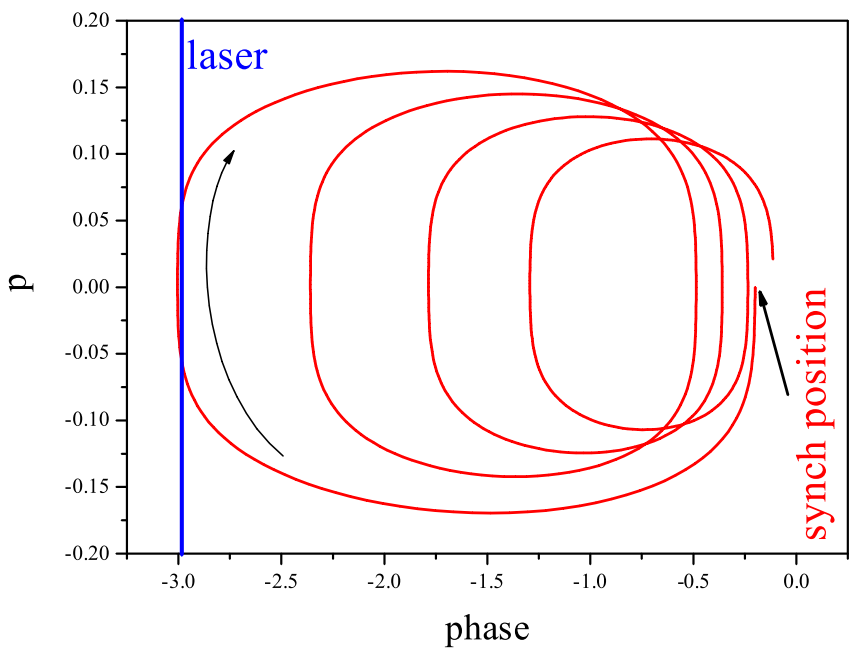

FIG. 3. (Color) Trajectory of the bunch centroid in RFPM mode (phase origin at zero of initial $\mathrm{rf}$ phase).

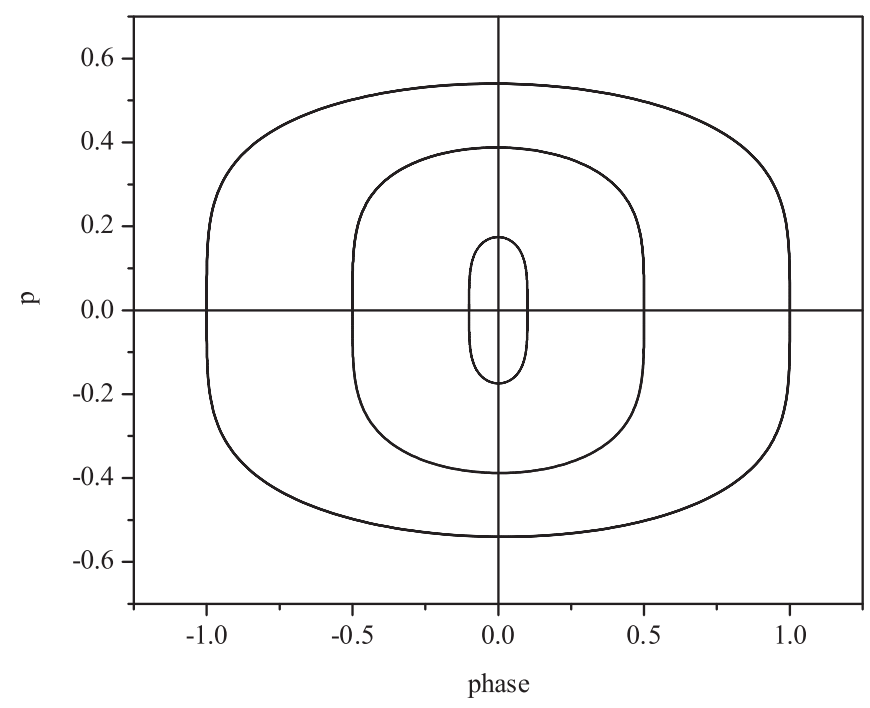

FIG. 4. The phase trajectory of the bunch center without excitation, damping, and RFPM.

the RFPM mode since here the curvature of the phase trajectory on which the electrons interact with the laser pulse can be made small with an appropriate value of the third-order momentum compaction, as is illustrated in Figs. 4 and 5. The synchrotron period in an optimized lattice (see Fig. 5) decreases with the amplitude.

Manipulation of the rf phase should bring the bunch into the left half of the phase plane where the interaction with the laser pulse pushes the particles back along the "straight" phase trajectory. As a result, the electrons are residing in the laser pulse for a longer period of time and scattering off more laser photons.

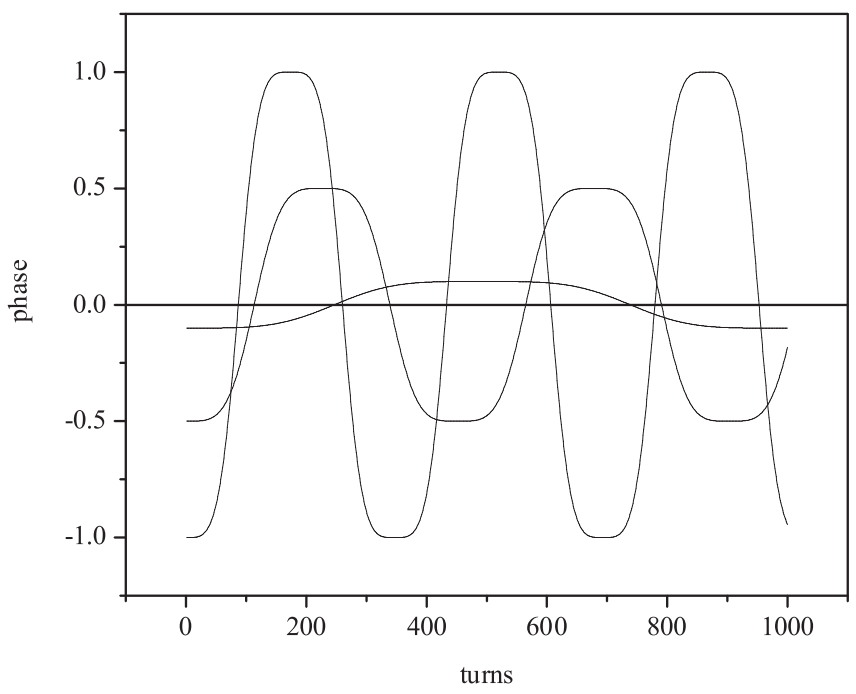

FIG. 5. The bunch center phase vs turn number for different initial phase displacements, without excitation, damping, and RFPM. 


\section{SIMULATIONS}

The analytical estimates of the steady-state distribution of electrons interacting with the laser pulse were based upon a linear smooth approximation and a Gaussian-noise perturbation. Specifically, the conventional analysis of the synchrotron-radiation dynamics splits the effects from the synchrotron-radiation emission into a viscous regular damping and a random excitation driven by additive noise (for the Compton ring the latter is negligible for the longitudinal motion if compared with the excitation from the Compton scattering), which is often treated as Gaussian white noise. In the same spirit, our above analysis has considered the recoil effects as multiplicative Gaussian noise, and treated the damping and excitation as uncorrelated processes. Integral recoils over a turn are of approximate Gaussian shape if many independent small-energy quanta are emitted. The Compton spectrum differs from a Gaussian significantly.

Also in other respects, the behavior of bunches circulating in a real gamma ring is more complex: a steady state may not be reached, the intensity of the interaction with the laser pulse depends on the particle coordinates at the interaction point, the laser and beam electron collide under a finite crossing angle and have finite pulse or bunch lengths, etc.

Against this background, simulations were carried out to verify the analytical predictions and to obtain a quantitative image of the bunch dynamics in a gamma ring.

The simulation code used for evaluating the longitudinal beam dynamics in gamma rings (see [1]) has been upgraded so as to incorporate the rf-phase manipulation mode and the transverse degrees of freedom as well.

In this code, the particle motion is simulated turn by turn. Every turn is represented by a nonlinear drift (with momentum compaction factors up to third order), a short rf cavity, a short longitudinal damping insert where the particle loses part of its energy due to the synchrotron radiation in arcs and wigglers, a short transverse damping and excitation insert providing the correct natural emittances without laser interaction, and the interaction point split into a number of slices. While passing a slice, the electron may experience an independent kick (scattering off a laser photon), the probability of which is determined by the local density of the laser pulse at the particle's coordinates.

In the model under consideration, the phase $\phi$ and the relative energy deviation $p$ when passing through the nonlinear "drift" and the rf cavity change as

$$
\begin{aligned}
& \phi_{f}=\phi_{i}+\left(\kappa_{1} p_{i}+\kappa_{2} p_{i}^{2}+\kappa_{3} p_{i}^{3}\right) ; \\
& p_{f}=p_{i}-U_{\mathrm{rf}} \sin \phi_{f}
\end{aligned}
$$

with $\kappa_{k}$ the phase slipping factors related to the momentum compaction factors $\alpha_{k}$ as

$$
\begin{aligned}
& \kappa_{1}=2 \pi h\left(\alpha_{1}-\gamma_{s}^{-2}\right) \\
& \kappa_{2}=2 \pi h\left(\alpha_{2}+\frac{3-2 \alpha_{1}}{2 \gamma_{s}^{2}}-\frac{1}{2 \gamma_{s}^{4}}\right) \\
& \kappa_{3}=2 \pi h\left[\alpha_{3}-\gamma_{s}^{-2}\left(2+\alpha_{2}-\frac{3}{2} \alpha_{1}\right)+\frac{1}{2 \gamma_{s}^{4}}\left(3-\alpha_{1}\right)\right],
\end{aligned}
$$

$h$ the harmonic number (number of rf buckets over the circumference), $U_{\mathrm{rf}}=e V_{\mathrm{rf}} / E_{b}\left(V_{\mathrm{rf}}\right.$ the rf voltage). Here used is expansion of the circumference in a form $L=$ $L_{0}\left(1+\alpha_{1} p+\alpha_{2} p^{2}+\alpha_{3} p^{3}\right)$.

The Compton kicks are randomly distributed in accordance with the ultrarelativistic or small-angle approximation (3), including a $\gamma^{2}$ dependence on the electron energy. The magnitude of the transverse deflections is determined from the total kick; the angle $\phi$ in Fig. 1 is random, uniformly distributed over $[0,2 \pi]$, while the angle $\psi$ obeys the two-particle kinematics. Because of interaction with a laser photon, the longitudinal "canonical momentum" $p$, and the transverse one $x^{\prime}$ (or $z^{\prime}$ ) transform as

$$
\begin{aligned}
& p_{f}=p_{i}(1-2 b \zeta)-b \zeta \\
& x_{f}^{\prime}=x_{i}^{\prime}(1-b \zeta)\left(1+p_{i}\right)+\frac{b}{\gamma_{s}} \sqrt{(1-\zeta) \zeta} \sin \psi,
\end{aligned}
$$

with $b=2 E_{\mathrm{las}}(1+\cos \varphi) \gamma_{s} / m_{e} c^{2}$. The vertical kicks are also described by (9b) with the substitution $\sin \psi \rightarrow \cos \psi$. The vertical kicks are described by (9b) with substitution $\sin \psi \rightarrow \cos \psi$.

Two simulations were performed. The first intended to verify the theoretical predictions for the steady state, and the second to simulate the ILC Compton gamma source in the pulsed mode.

Simulations of the steady-state parameters for a bunch circulating in a Compton ring (with moderate energy) have verified the theoretical predictions: (i) the partial Compton emittances are independent of the frequency of electronphoton interactions; (ii) the simulated energy spread is in agreement with estimates in which the recoil momenta are assumed to be random without systematic component, even though in the simulation the random perturbations are not centered; (iii) the total emittances due to the combined effect of Compton scattering, synchrotron damping, and quantum excitation agree with the theoretical predictions; (iv) the transition periods to the steady state obey the Kolomenskij-Lebedev-Robinson theorem with the vertical and horizontal betatron damping times equal to each other, and 2 times shorter than the longitudinal synchrotron damping time; (v) the energy spread in an essentially nonlinear lattice in the main stable area (around zero phase) is in good agreement with that predicted for the lattice with linear momentum compaction factor (5).

For simulations of the gamma source, two lattices were taken: ILC YAG $n l$ lattice [1], and CLIC (main parameters are listed in Table II). The emittances together with the 
TABLE II. Main parameters of models.

\begin{tabular}{lcc}
\hline \hline \multicolumn{1}{c}{ Model } & CLIC & ILC YAG \\
\hline Electrons energy $E_{b}(\mathrm{GeV})$ & 1.301 & 1.301 \\
rf frequency $E_{b}(\mathrm{MHz})$ & 1875 & 650 \\
Harmonic number $h$ & 840 & 600 \\
rf voltage $V_{\text {rf }}(\mathrm{MV})$ & 20 & 20 \\
Compaction $\alpha_{1}$ & $5 \times 10^{-5}$ & $2 \times 10^{-6}$ \\
Compaction $\alpha_{2}$ & 0 & 0 \\
Compaction $\alpha_{3}$ & $2 \times 10^{-3}$ & $2 \times 10^{-3}$ \\
Natural emittance $\epsilon_{x}(\mathrm{~m} \mathrm{rad})$ & $2.1 \times 10^{-8}$ & $1 \times 10^{-9}$ \\
Natural emittance $\epsilon_{z}(\mathrm{~m} \mathrm{rad})$ & $1.05 \times 10^{-9}$ & $5 \times 10^{-11}$ \\
Natural losses $\Delta E_{\mathrm{sr}}(\mathrm{MeV})$ & 0.21 & 0.65 \\
Full cycle (turns) & 14888 & 10833 \\
Laser on/off (turns) & $1 / 2546$ & $68 / 168$ \\
Power in ip cavities $(\mathrm{J})$ & 0.592 & 17.76 \\
Laser-photon energy $E_{\text {las }}(\mathrm{eV})$ & 1.164 & 1.164 \\
Laser rms pulse length $(\mathrm{mm})$ & 0.9 & 0.9 \\
Laser rms pulse radius $(\mu \mathrm{m})$ & 5 & 5 \\
Crossing angle $\varphi(\mathrm{rad})$ & 0.13963 & 0.13963 \\
Maximum yield $(\mathrm{gamma} / \mathrm{electron} /$ turn $)$ & 0.11 & 3.2 \\
\hline \hline
\end{tabular}

betatron amplitudes at the IP, $\beta_{\text {ip }}$, were set such that the partial Compton radial emittance was smaller than the synchrotron one, while the vertical one was larger: at $\beta_{x}^{(\mathrm{ip})}=\beta_{z}^{(\mathrm{ip})}=0.5 \mathrm{~m}$ the partial Compton emittances were $\epsilon_{x}^{(\mathrm{C})}=\epsilon_{z}^{(\mathrm{C})}=1.35 \times 10^{-10} \mathrm{~m} \mathrm{rad}$, and the natural (synchrotron) emittances $\epsilon_{x}=1 \times 10^{-9} \mathrm{~m} \mathrm{rad}, \epsilon_{z}=5 \times$ $10^{-11} \mathrm{~m} \mathrm{rad}$.

The amplitude of rf-phase deviation for RFPM mode was chosen such that the bunch phase at the beginning of interaction with the laser pulse became -1.5 from the plateau, which provided the maximal yield. The synchro-

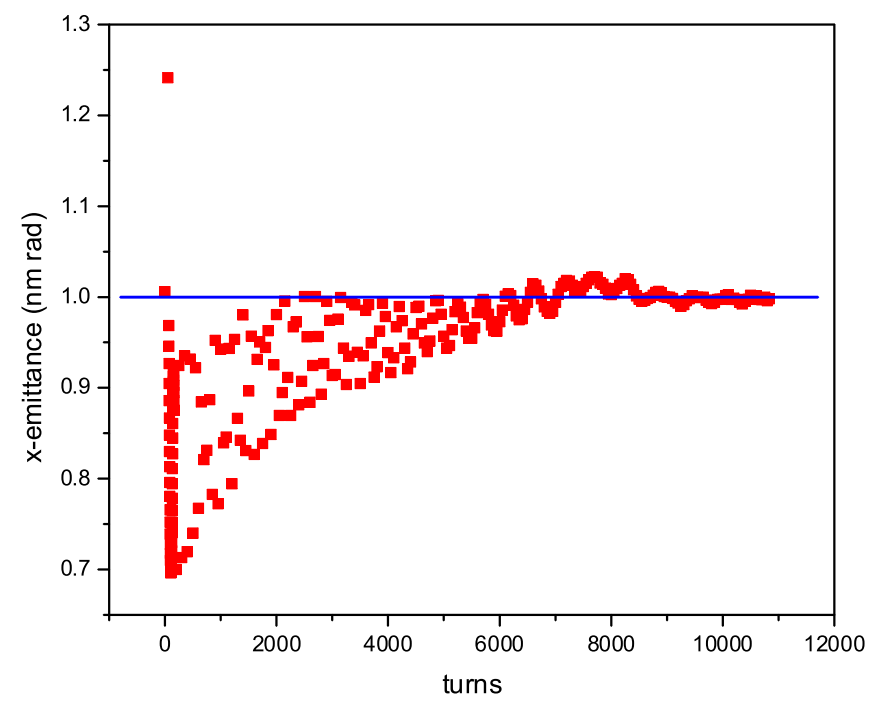

FIG. 6. (Color) Simulated horizontal emittance variation with turn number. The blue lines indicate the natural (synchrotron) emittances. The laser pulse is matched to the synchronous particle.

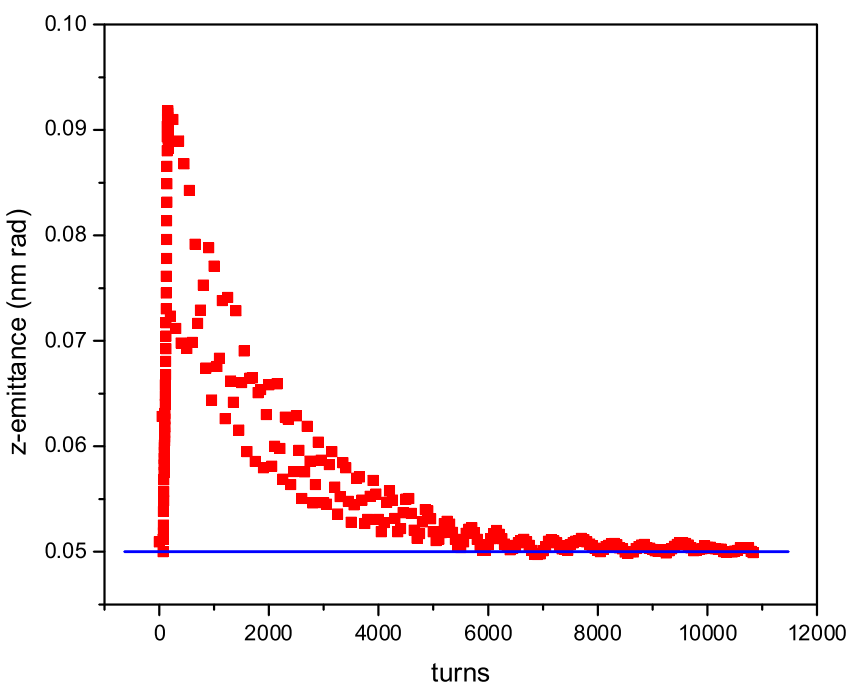

FIG. 7. (Color) Simulated vertical emittance variation with turn number.

tron period for this amplitude was 92 turns (without laser interactions and synchrotron losses).

Results of the simulations are presented in Figs. 6 and 7, which show that the horizontal emittance cools down due to the laser interaction, while the vertical one increases. The oscillations in the emittances are caused by the coherent synchrotron oscillations inherent in the rf-phase manipulation mode, possibly related to the temporary adiabatic damping or antidamping of betatron oscillations.

Results of a 3D simulation of repeated working cycles in the ILC YAG Compton ring for the pulsed mode with and without rf-phase manipulation are consistent with those presented in [1], which were obtained for an idealized 1D model.

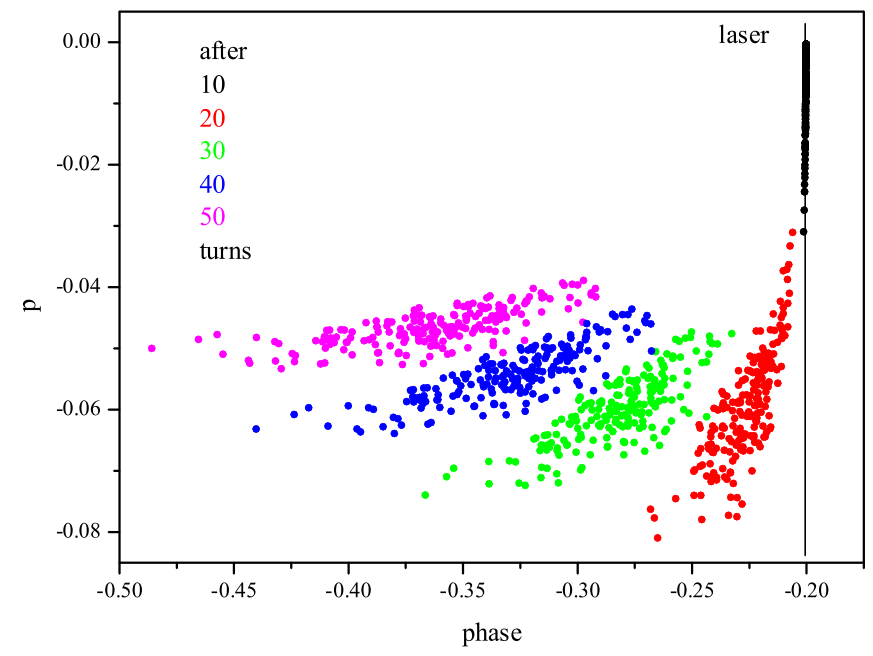

FIG. 8. (Color) Snapshots of macroparticle distributions during the interaction with an intense laser pulse matched with the synchronous particle, for the ILC YAG Compton ring (phase origin at zero of the initial rf phase). 


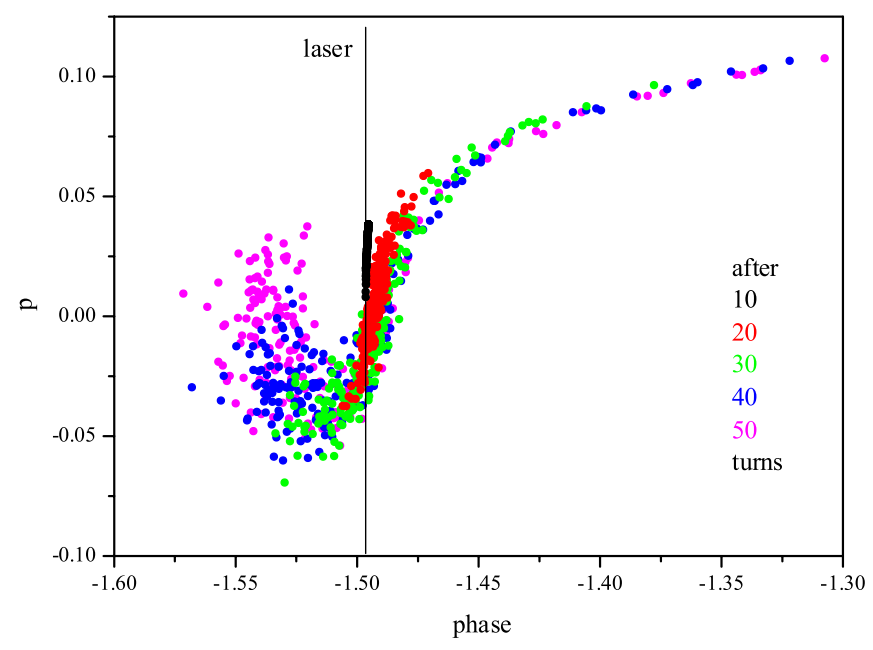

FIG. 9. (Color) Snapshots of macroparticle distributions during the interaction with an intense laser pulse in the rf-phase manipulation mode, for the ILC YAG Compton ring (phase origin at zero of the plateau rf phase).

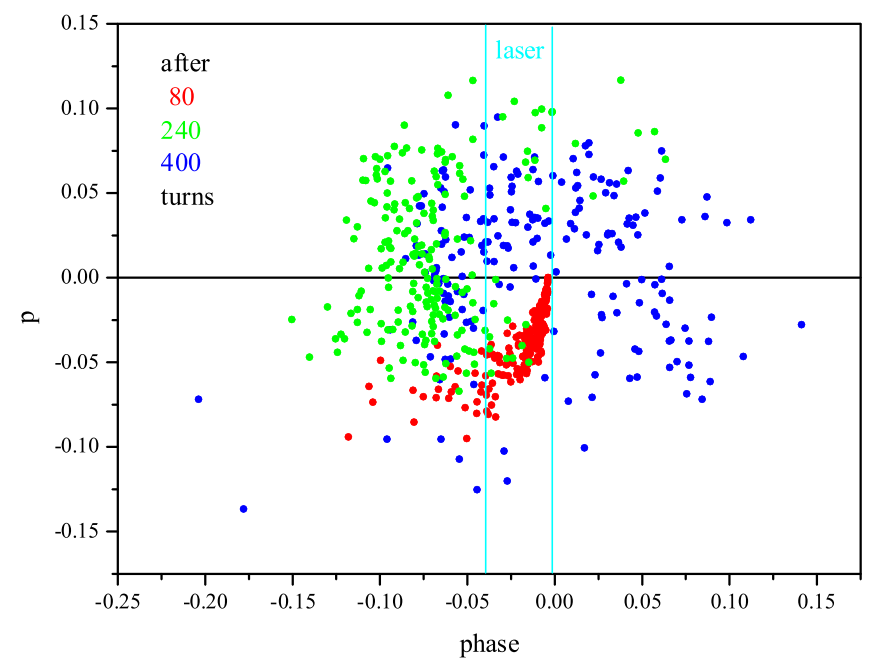

FIG. 10. (Color) Snapshots of macroparticle distributions during the interaction with the laser pulse in the CLIC YAG Compton ring (phase origin at zero of the initial rf phase).

The ILC model produces about 67 gammas per electron per 100 turns in RFPM mode as compared with 20 gammas in the mode without the phase manipulation. Also a significant attenuation of the phase velocity due to the interactions with the laser pulse was noticed: with the synchrotron period (92 turns) shorter than the laser pulse (100 turns) the vast part of the bunch remained in synchronism with the laser during the pulse.

In Figs. 8-10, the simulated distributions of macroparticles in the longitudinal phase space are presented for the ILC and CLIC YAG rings.

The snapshots manifest differences in the particle kinetics for the nonlinear lattice. They also demonstrate the advantage of the phase manipulation scheme. The CLIC
Compton ring with a total laser-pulse photon population equal to $1 / 30$ th of ILC's can yield much longer gamma trains (of lower intensity).

The laser-induced transverse kinetics of electron bunches circulating in the Compton gamma sources does not deteriorate the performance of these sources compared with the one estimated without account of the transverse degrees of freedom: With the proper small beta functions at the interaction point, the transverse natural emittances may even be reduced due by the beam-laser interaction.

\section{SUMMARY AND OUTLOOK}

The single-particle dynamics of electron bunches in Compton gamma sources has been studied analytically and in simulations. The analytical estimates for the steady-state bunch parameters are in good agreement with those obtained from the simulations.

Our study confirms that the interaction of the electrons with the laser photons does not significantly affect the transverse degrees of freedom. The bottleneck is the longitudinal dynamics: the ring's energy acceptance should be unusually large.

Further studies should comprise multiparticle dynamics (collective effects) in view of the required large bunch population. Also, methods for attaining short bunches will be developed.

\section{ACKNOWLEDGMENTS}

The authors would like to thank the participants of the POSIPOL 06 workshop [17] for fruitful discussions on the gamma sources.

[1] S. Araki, Y. Higashi, Y. Honda, Y. Kurihara, M. Kuriki, T. Okugi, T. Omori, T. Taniguchi, N. Terunuma, J. Urakawa, X. Artru, M. Chevallier, V. Strakhovenko, E. Bulyak, P. Gladkikh, K. Mönig, R. Chehab, A. Variola, F. Zommer, S. Guiducci, P. Raimondi, F. Zimmermann, K. Sakaue, T. Hirose, M. Washio, N. Sasao, H. Yokoyama, M. Fukuda, K. Hirano, M. Takano, T. Takahashi, H. Sato, A. Tsumeni, J. Gao, and V. Soskov, physics/0509016, 2005.

[2] F. Zimmermann, H. Braun, M. Korostelev, L. Rinolfi, D. Schulte, E. Bulyak, P. Gladkikh, S. Araki, Y. Higashi, Y. Honda, Y. Kurihara, M. Kuriki, T. Okugi, T. Omori, T. Taniguchi, N. Terunuma, J. Urakawa, K. Mönig, A. Variola, F. Zommer, X. Artru, R. Chehab, M. Chevallier, V. Strakhovenko, J. Gao, S. Guiducci, P. Raimondi, V. Soskov, M. Fukuda, K. Hirano, M. Takano, T. Hirose, K. Sakaue, M. Washio, T. Takahashi, H. Sato, and A. Tsumeni, in EPAC 2006, Edinburgh, Scotland, 2006, http://oraweb.cern.ch/pls/epac06/toc.htm/WEPLS060.pdf (2006).

[3] Z. Huang and R. D. Ruth, Phys. Rev. Lett. 80, 976 (1998). 
[4] B. Barish, in EPAC 2006, Edinburgh, Scotland, 2006, http://accelconf.web.cern.ch/accelconf/e06/ PAPERS/MOXPA01.pdf (2006).

[5] A. Ghigo, in EPAC 2006, Edinburgh, Scotland, 2006, http://accelconf.web.cern.ch/accelconf/e06/ PAPERS/WEYPA03.pdf (2006).

[6] E. Bulyak, P. Gladkikh, A. Zelinsky, I. Karnaukhov, S. Kononenko, V. Lapshin, A. Mytsykov, Yu. Telegin, A. Khodyachikh, A. Shcherbakov, V. Molodkin, V. Nemoshkalenko, and A. Shpak, Nucl. Instrum. Methods Phys. Res., Sect. A 487, 241 (2002).

[7] R. J. Loewen, Ph.D. thesis, Stanford, 2003.

[8] J. Urakawa, K. Kubo, N. Terunuma, T. Taniguchi, Y. Yamazaki, K. Hirano, M. Nomura, I. Sakai, M. Takano, N. Sasao, E. Bulyak, P. Gladkikh, A. Mytsykov, A. Zelinsky, and F. Zimmermann, Nucl. Instrum. Methods Phys. Res., Sect. A 532, 388 (2004).

[9] E. Bulyak, in Proceedings of the EPAC-2004 (Luzern, Switzerland), http://accelconf.web.cern.ch/accelconf/e04/ papers/thpkf063.pdf (2004).

[10] E. Bulyak and V. Skomorokhov, in Proceedings of the
EPAC-2004 (Luzern, Switzerland), http://accelconf.web. cern.ch/accelconf/e04/papers/weplt138.pdf (2004).

[11] G. Budker and S. Belyaev, in Plasma Physics and the Problem of Controllable Fusion, Vol. II (USSR Academy of Science, Moscow, 1958), in Russian.

[12] K. L. Brown, F. Rothacker, D. C. Carey, and F. Iselin, Technical Report SLAC-R-91, Rev. 2, SLAC, 1977.

[13] K. Floettmann, Phys. Rev. ST Accel. Beams 6, 034202 (2003).

[14] S. Y. Lee, Accelerator Physics (World Scientific, Singapore, 1999).

[15] J. Feikes, K. Holldack, P. Kuske, G. Wüstefeld, and H.-W. Hübers, ICFA Beam Dynamics Newsletter 35, 82 (2004), http://icfa-usa.jlab.org/archive/newsletter/ icfa_bd_nl_35.pdf.

[16] E. Bulyak, P. Gladkikh, and V. Skomorokhov, physics/ 0505204, 2005.

[17] Workshop on polarized positron sources for ILC and CLIC-POSIPOL, http://posipol2006.web.cern.ch/ Posipol2006 (2006). 